Sposito, L.A.C.; Nakamura, P.M.; Monteiro, H.L.; Papini, C.B.; Benedetti, T.R.B.; Kokubun, E. (2021). Evaluation of Strategies for the Physical Activity Promotion: Cost-Utility Study. Revista Internacional de Medicina y Ciencias de la Actividad Física y el Deporte vol. 21 (84) pp. 607-621. Http://cdeporte.rediris.es/revista/revista83/artevaluacion1269.htm

DOI: https://doi.org/10.15366/rimcafd2021.83.012

\title{
ORIGINAL
}

\section{EVALUACIÓN DE ESTRATEGIAS PARA LA PROMOCIÓN DE LA ACTIVIDAD FÍSICA: COSTO- UTILIDAD}

\section{EVALUATION OF STRATEGIES FOR THE PHYSICAL ACTIVITY PROMOTION: COST-UTILITY STUDY}

Sposito, L.A.C. 1; Nakamura, P.M. 2; Monteiro, H.L. ${ }^{3}$; Papini, C.B.4; Benedetti, T.R.B. ${ }^{5}$ y Kokubun, E. ${ }^{6}$

\footnotetext{
1 Posgrado en Ciencias de la Motricidad de la Universidad Estadual Paulista Júlio de Mesquita Filho - UNESP (Brasil) sposito.ef@gmail.com

2 Profesora Doctora del Instituto Federal de Educación, Ciencia y Tecnología del Sur de Minas Gerais, Campus Muzambinho - IFSULDEMINAS (Brasil) missaki.naka@gmail.com

${ }^{3}$ Profesor Asistente Doctor de la Universidad Estadual Paulista Júlio de Mesquita Filho - UNESP (Brasil) heu@fc.unesp.br

4 Profesora Adjunta Doctora de la Universidad Federal do Triângulo Mineiro - UFTM (Brasil) mila_papini@yahoo.com.br

5 Profesora Adjunta Doctora de la Universidad Federal de Santa Catarina - UFSC (Brasil) tania.benedetti@ufsc.br

${ }^{6}$ Profesor Titular Doctor de la Universidad Estadual Paulista Júlio de Mesquita Filho - UNESP (Brasil) ekokubun@gmail.com
}

\section{AGRADECIMIENTOS O FINANCIACIÓN}

Este artículo no presenta conflicto de intereses ante otras entidades académicas. Este proyecto se pudo desarrollar gracias al apoyo económico generado por la CAPES.

Código UNESCO / UNESCO code: 3212 . Salud Pública / Public Health Clasificación del Consejo de Europa / Council of Europe classification: 17. Otras: Actividad Física y Salud/Others: Physical Activity and Health

Recibido 8 de julio de 2019 Received July 8, 2019

Aceptado 29 de febrero de 2020 Accepted February 29, 2020

\section{RESUMEN}

El objetivo fue comparar el costo adicional por Años de Vida Ajustados por Calidad (AVAC) de tres estrategias para promoción de Actividad Física (AF) y verificar la influencia de las intervenciones sobre el porcentaje de adultos físicamente activos en las Unidades de Salud Básicas (USB). Los usuarios de 
las USB fueron seleccionados y distribuidos en grupos aleatorizados: (1) breve asesoramiento - recibió orientaciones para aumentar la AF; (2) grupo de indicación al programa de ejercicio físico supervisado - invitados a participar en programa de ejercicio físico en la USB; y, (3) grupo evaluación - no recibió ninguna intervención, respondiendo a los cuestionarios propuestos por la investigación. Los grupos fueron entrevistados en cinco momentos, durante un año, evaluados sobre el costo por AVAC ganado y tiempo de AF. La estrategia de breve asesoría fue más efectiva, comparada a las demás estrategias, debido al menor costo/AVAC y resultados positivos sobre la AF.

PALABRAS CLAVE: Actividad Física, Asesoramiento, Atención Primaria en Salud y Brasil.

\section{ABSTRACT}

The aim was to compare the additional cost by Quality Adjusted Life Years (QALY) gained from three strategies for physical activity promotion and verify the interventions influence on the percentage of physically active adults in Basic Health Units (BHU). BHU users were randomized into three groups: (a) brief counseling - received guidelines to increase physical activity (PA) time; (b) indication group to the supervised physical exercise program - was invited to participate in the physical exercise program at the BHU; and, (c) evaluation group - didn't receive any type of intervention and only answered the questionnaires proposed by the research. The individuals in the three groups were interviewed in five moments, during a year, being evaluated on the cost by QALY gained and PA time. The strategy of brief counseling was more effective when compared to the other strategies, due to the lower cost/QALY and indication of positive results on PA.

KEY WORDS: Physical Activity, Counseling, Primary Health Care and Brazil.

\section{INTRODUCCIÓN}

Se estima que el $31 \%$ de la población mundial y el $45.1 \%$ de los brasileños no llegan a cumplir las recomendaciones de práctica de actividad física ${ }^{1,2}$. El uso de estrategias para fomentar la práctica de actividad física en el sistema público de Atención Primaria de Salud es adoptado por muchos países desarrollados como una forma de combatir la inactividad física ${ }^{3}$. En Brasil, en el área de la salud, hay un aumento significativo en el número de programas comunitarios, entre ellos, la provisión de clases de ejercicio físico supervisadas en unidades de salud básicas y programas de asesoramiento ${ }^{4}$; sin embargo, hay una escasez de investigaciones sobre el Costo-utilidad de estas acciones.

El análisis de costo-utilidad promueve una unidad de medida llamada AVAC, conocida como los años de vida ajustados por calidad, en la que incorpora tanto la calidad de vida (morbilidad) como la cantidad de vida (mortalidad). Los datos se pueden obtener mediante algunas técnicas o cuestionarios validados para el país de aplicación. De esa manera, al calcular el costo de la 
intervención/procedimiento de salud más la variable AVAC, es posible realizar el análisis de costo-utilidad. Este tipo de análisis se utiliza para comparar y verificar la efectividad entre dos o más intervenciones/procedimientos de salud ${ }^{5}$.

Algunos estudios en países desarrollados han demostrado la efectividad de la estrategia de asesoramiento breve para la práctica de la actividad física en comparación con el ejercicio físico supervisado, especialmente relacionado con el costo-utilidad ${ }^{6,7}$. Países, como Reino Unido, Nueva Zelanda y Australia han adoptado la preferencia de asesoramiento breve en Atención Primaria de Salud debido a su efectividad en el aumento del tiempo de actividad física, produciendo mayores ganancias en Años de Vida Ajustados por Calidad (AVAC) y siendo más económicos para el sector de la salud?

La asesoría busca fomentar conductas saludables como el abandono del hábito de fumar, la alimentación saludable y actividad física, a través de sesiones individuales o grupales, que duran de cinco a treinta minutos ${ }^{8,9}$. En Brasil, se considera una estrategia educativa prevista por la política nacional de promoción de la salud ${ }^{10}$. A pesar de esto, todavía se usa poco como recurso para la promoción de actividad física a usuarios de Atención Primaria de Salud brasileños ${ }^{11,12,13}$.

Hasta la fecha, no hay información sobre el costo por AVAC obtenido en relación con las estrategias de asesorías cortas para la práctica de la actividad física, los programas de ejercicio supervisado y la estrategia de evaluación de Atención Primaria de Salud. Además, el acceso a los sistemas de salud y el consumo de servicios varía de un lugar a otro, y es importante proporcionar una evaluación económica que aborde la realidad de cada contexto, especialmente orientadas a estrategias de promoción de la salud.

Por lo tanto, el objetivo de este estudio fue comparar el costo adicional por AVAC obtenido de tres estrategias diferentes para promover la actividad física en las Unidades de Atención Primaria de Salud y verificar la influencia de estas intervenciones en el porcentaje de adultos físicamente activos.

\section{METODOLOGÍA}

Diseño

Se trata de un estudio aleatorizado, sin doble ciego, realizado en Unidades de Salud Básicas (USB) ubicadas en la región sudeste de la ciudad de Rio ClaroSão Paulo, Brasil. El municipio tiene 186.253 habitantes, dispone de 22 USB y posee Índice de Desarrollo Humano de 0.803. Para esta investigación, fueron seleccionadas, por conveniencia, cuatro USB con profesionales de Educación Física, vinculados al Programa de Estrategia de Salud de la Familia y asistidas por el Núcleo de Atención a la Salud de la Familia.

Esta investigación fue aprobada por el Comité de Ética de Investigaciones con Seres Humanos del Instituto de Biociencias de la UNESP-Rio Claro (São PauloBrasil), con el protocolo número 1.548.264. Después de terminar el estudio, los 
participantes del grupo evaluación fueron invitados por teléfono a participar del Programa de Ejercicios Físicos en su respectiva USB.

Reclutamiento de la Muestra

Los invitados a esta encuesta son usuarios del Sistema Único de Salud, adultos de ambos géneros. Se incluyeron del estudio individuos de edad $\geq 18$ años y con registro de salud activo por lo menos de dos años. Para los criterios de exclusión del estudio se consideró: no cumplir a tres entrevistas pre-programadas y personas con discapacidades intelectuales y físicas.

Para el contacto presencial con los participantes se cumplieron las siguientes etapas: a) autorización de la Secretaría Municipal de Salud para realización de la investigación; b) autorización de los coordinadores de las USB para iniciar los trabajos; c) sorteo de los prontuarios activos por la función aleatoria del programa Excel ${ }^{\circledR}$ y automáticamente la aletorización de las intervenciones: (1) Breve Asesoramiento, (2) Indicación para práctica de Ejercicio Físico en la USB y (3) Grupo Evaluación; d) análisis de registros para la recolección de teléfonos de los usuarios; e) intento de contacto telefónico con los usuarios a fin de invitar a participar en la investigación y la programación de la entrevista presencial.

\section{Intervenciones}

Breve Asesoramiento (BA): los usuarios que participaron en este grupo, cuyo objetivo fue incentivar la práctica de actividad física, asistieron a la USB cada tres meses, cumpliendo cinco diferentes momentos de consejería durante un año. Cada sesión de BA duró, en promedio 20 minutos, y fue realizado individualmente por los investigadores. Se abordaron conceptos sobre: 1. Y ¿Qué es actividad física, sus dominios y beneficios?; 2. ¿Qué es el comportamiento sedentario, sus riesgo y cómo reducirlo?; 3. ¿Cuáles la importancia de alcanzar las recomendaciones en actividad física, aprendiendo a organizar el tiempo y la alimentación sana?; 4. ¿Cuáles son las intensidades de actividad física y las capacidades físicas recomendadas que pueden proporcionar mayores beneficios a la salud?; y, 5. Transponiendo barreras y estableciendo metas reales para la práctica de actividad física y los lugares para desarrollar prácticas gratuitas en el barrio ${ }^{14}$. Esta información fue transmitida por los investigadores a través de 5 folletos ilustrativos.

La indicación para la práctica de Ejercicio Físico en la USB (IPEF-USB): los usuarios fueron invitados a participar del programa de Ejercicio Físico, ofrecido regularmente en las USB de Río Claro (São Paulo, Brasil). Las clases eran de carácter generalizado y de intensidad leve a moderada, realizándose durante dos días alternados por semana, con una duración de 60 minutos y siempre bajo la orientación de un profesional de la educación física. El objetivo de esta intervención, además de aumentar el nivel de actividad física, es desarrollar la aptitud física general a partir de ejercicios de fuerza, flexibilidad, agilidad, capacidad aeróbica y coordinación motora, así como, promover calidad de vida a los participantes ${ }^{15}$. 
El Grupo Evaluación (GE): estos usuarios no recibieron asesoramiento para la práctica de actividad física, siendo invitados sólo para realizar las evaluaciones.

Instrumentos de Evaluación

Las evaluaciones fueron realizadas en los tres grupos (BA, IPEF-USB y GE) en cinco momentos, con frecuencia cada tres meses, totalizando 1 año de las intervenciones, cuyo inicio fue en enero de 2016.

\section{Cuestionario de Perfil}

A partir de una entrevista a través del cuestionario, se presentó el perfil del participante, sobre: edad, sexo, trabajo, enfermedades, tabaquismo, uso de bebidas alcohólicas, hábitos alimentarios y nivel de estrés.

\section{Medidas Antropométricas y de la Composición Corporal}

La estatura y el peso se midieron por medio de una báscula antropométrica (marca FILIZOLA®, Brasil). A partir de estas informaciones se calculó el Índice de Masa Corporal. Para la clasificación de los individuos, según condición nutricional, se utilizaron los siguientes intervalos: bajo peso $\left(<18.5 \mathrm{~kg} / \mathrm{m}^{2}\right)$, peso normal $\left(18.5-24.9 \mathrm{~kg} / \mathrm{m}^{2}\right)$, sobrepeso $\left(25-29.9 \mathrm{~kg} / \mathrm{m}^{2}\right)$ y obesidad $\left(\geq 30 \mathrm{~kg} / \mathrm{m}^{2}\right)$.

\section{Años de Vida Ajustados por Calidad (AVAC)}

Para la obtención del AVAC se utilizó el cuestionario SF-36, traducido y validado para Brasil ${ }^{16}$. El instrumento está compuesto por ocho elementos que evalúan la calidad de vida. Para el cálculo del AVAC, seis áreas se consideraron, como: capacidad funcional, capacidad física y psicológica, dominio social, dominio dolor, dominio mental y dominio de vitalidad.

De esta forma, a partir de una base de datos con los valores del SF-36 se aplicó el algoritmo producido en Brasil ${ }^{17}$ para obtener los valores utilitarios, en el cual se adquirió la autorización de los investigadores por parte del Instituto de Evaluación de Tecnología de Salud (IATS).

El valor utilitario es una cuantificación de la calidad de vida del individuo en un solo valor que varía de 0 a 1 . El valor de utilidad 0 representa el peor estado de salud o muerte y el valor 1 corresponde a la salud perfecta /calidad plena, que se utiliza como medida de desenlace en análisis de costo-utilidad ${ }^{17}$.

El cálculo del AVAC se realizó multiplicando el valor utilitario por el tiempo de duración de la intervención. La medida de supervivencia de este estudio se consideró a partir de la proporción del intervalo de duración entre una evaluación y otra, sobre la duración total del estudio ${ }^{18}$. 
Costos de las Intervenciones

Los costos de las intervenciones se basaron en los gastos resarcidos por el Sistema Único de Salud, y se apoyaron en tres parámetros: valor hora de clase del profesional de educación física, costo local y material de clase (carpeta para el BA y balón para el IPEF-USB), excepto la intervención GE que consideró sólo las dos primeras variables.

\section{La Razón Costo-Efectividad Incremental (RCEI)}

El cálculo del RCEI permite medir la efectividad/costo-utilidad de diferentes estrategias, siendo asociado el costo incremental por una unidad de efecto, en este caso medida en AVAC. Para el cálculo del RCEI se utilizó la ecuación abajo $^{19}$ :

\section{Ecuación 1:}

RCEI = costo de la intervención nueva - costo de la intervención estándar

AVAC de la nueva intervención - AVAC del estándar

Medición de la Actividad Física y Comportamiento Sedentario

Se utilizó el International Physical Activity Questionnaire (IPAQ-versión larga) para verificar el tiempo de actividad física y tiempo de comportamiento sedentario referente a la última semana.

Se calculó el tiempo en minutos de actividad física semanal en los cuatro dominios a partir de la ecuación ${ }^{20}$ : Actividad Física $=$ caminata + bicicleta + actividad física moderada $+(2 \times$ actividad física vigorosa). Después del cálculo, los individuos con valores $>150 \mathrm{~min} / \mathrm{semana}$ fueron considerados físicamente activos. Para esta investigación fueron consideradas la actividad física en el tiempo libre, transporte y tiempo total (suma del tiempo de actividad física en el tiempo libre, transporte, trabajo y medio doméstico).

\section{Análisis Estadístico}

Para los datos del presente estudio se adoptó el análisis de la intención de tratar y demostrados con media, desviación estándar y distribución porcentual por categoría de respuesta. Se aplicó ANOVA one-way con post hoc de Tukey para la comparación de promedios entre los grupos sobre las variables edad, escolaridad, Índice de Masa Corporal, tiempo sentado y tiempo de actividad física.

Para las variables demográficas y de salud se realizó el conteo de frecuencias observadas y se aplicó la prueba de chi cuadrado con el objetivo de identificar la diferencia de proporción entre los sexos sobre los grupos BA, IPEF-USB y GE. Para verificar posibles diferencias estadísticas entre las clasificaciones $(0$ a 10 $\mathrm{min} / \mathrm{sem}),(11$ a $149 \mathrm{~min} / \mathrm{sem})$ y $(\geq 150 \mathrm{~min} / \mathrm{sem})$ y los grupos en diferentes 
momentos (meses) para la actividad física en el tiempo libre, en el transporte y el tiempo total se utilizó la prueba chi-cuadrado.

Todos los análisis se realizaron a través del Statistical Package for the Social Sciences ${ }^{\circledR}$ (SPSS) versión 21 (IBM) adoptando $p<0,05$.

\section{RESULTADOS}

En el estudio participaron 75 usuarios, siendo $68 \%$ mujeres con edad promedio de $50 \pm 14$ años, ingreso promedio de $\$ 432.84$ e Índice de Masa Corporal de $28.34 \pm 6.37 \mathrm{~kg} / \mathrm{m}^{2}$.

La tabla 1 presenta el perfil de los grupos estudiados, de acuerdo con la edad, escolaridad, Índice de Masa Corporal, tiempo sentado y tiempo de actividad física en el tiempo libre, transporte y total. No se encontraron diferencias estadísticamente significativas entre los grupos en el momento previo a la intervención.

Tabla 1. ANOVA one-way y perfil de los usuarios de la Atención Primaria de Salud en el momento inicial de la investigación (Rio Claro - SP, $n=75$ )

\begin{tabular}{lcccc}
\hline \multicolumn{1}{c}{$\begin{array}{c}\text { Perfil } \\
\text { Media } \pm \text { DE }\end{array}$} & $\begin{array}{c}\text { BA } \\
(\mathbf{n}=\mathbf{3 6})\end{array}$ & $\begin{array}{c}\text { IPEF-USB } \\
(\mathbf{n}=\mathbf{2 1})\end{array}$ & $\begin{array}{c}\text { GE } \\
(\mathbf{n}=18)\end{array}$ & p \\
\hline Edad (años) & $50 \pm 14.82$ & $51 \pm 8.74$ & $50 \pm 19.76$ & 0.98 \\
Escolaridad (años) & $5.5 \pm 2.80$ & $5.3 \pm 3.18$ & $4.7 \pm 3.43$ & 0.66 \\
$\begin{array}{l}\text { Índice de Masa } \\
\text { Corporal (kg/m²) }\end{array}$ & $28.40 \pm 6.73$ & $28.25 \pm 4.15$ & $28.34 \pm 7.97$ & 0.99 \\
$\begin{array}{l}\text { Tiempo sentado (horas } \\
\text { y min/día) }\end{array}$ & $2 \mathrm{~h} 09 \pm 0.44$ & $2 \mathrm{~h} 20 \pm 0.54$ & $2 \mathrm{~h} 43 \pm 1.34$ & 0.17 \\
$\begin{array}{l}\text { Actividad física en } \\
\text { Tiempo libre } \\
\text { (min/sem) }\end{array}$ & $95.81 \pm 190.80$ & $68.19 \pm 106.67$ & $55.83 \pm 172.48$ & 0.69 \\
$\begin{array}{l}\text { Actividad física en } \\
\text { Transporte (min/sem) }\end{array}$ & $141.64 \pm 189.95$ & $99.95 \pm 88.08$ & $90.56 \pm 137.42$ & 0.51 \\
$\begin{array}{l}\text { Actividad física Total } \\
\text { (min/sem) }\end{array}$ & $1064.30 \pm 974.14$ & $946.80 \pm 825.06$ & $806.67 \pm 844.22$ & 0.61 \\
\hline
\end{tabular}

Leyenda: BA - Breve asesoramiento; IPEF-USB - Indicación para la práctica de ejercicio físico en unidades de salud básicas; GE - Grupo de evaluación; min/día - minutos al día; min/sem - minutos a la semana; DE desviación estándar; $p<0.05$

Los resultados de la tabla 2, indican que los participantes de cada grupo son representados predominantemente por mujeres, con elevada prevalencia de Enfermedades no Transmisibles (ENT) y estrés moderado a alto. Para las variables evaluadas, no se encontraron diferencias estadísticamente significativas entre los grupos. 
Tabla 2. Información de perfil de los usuarios de USB en el momento inicial de la investigación,

y sus resultados de la prueba de chi cuadrado (Rio Claro - SP, $n=75$ )

\begin{tabular}{|c|c|c|c|c|}
\hline $\begin{array}{l}\text { Perfil } \\
\text { (n) \% }\end{array}$ & $\begin{array}{c}\text { BA } \\
(n=36)\end{array}$ & $\begin{array}{c}\text { IPEF-USB } \\
(n=21)\end{array}$ & $\begin{array}{c}\text { GE } \\
(n=18)\end{array}$ & $\mathbf{p}$ \\
\hline Sexo Femenino & (24) 66.6 & (14) 66.6 & (13) 72.2 & \\
\hline Sexo Masculino & (12) 33.4 & (7) 33.4 & (5) 27.8 & 0.90 \\
\hline \multicolumn{5}{|l|}{ Trabajo remunerado } \\
\hline $\mathrm{Si}$ & (19) 52.8 & (10) 47.6 & (9) 50.0 & \multirow{2}{*}{0.93} \\
\hline No & (17) 47.2 & (11) 52.4 & (9) 50.0 & \\
\hline \multicolumn{5}{|l|}{ Personas con ENT } \\
\hline $\mathrm{Si}$ & (24) 66.7 & (14) 66.7 & (9) 50.0 & \multirow{2}{*}{0.44} \\
\hline No & (12) 33.3 & (7) 33.3 & (9) 50.0 & \\
\hline \multicolumn{5}{|l|}{ Fumadores } \\
\hline $\mathrm{Si}$ & (4) 11.1 & (7) 33.3 & (3) 16.7 & \multirow{2}{*}{0.11} \\
\hline No & (32) 88.9 & (14) 66.6 & (15) 83.3 & \\
\hline \multicolumn{5}{|l|}{ Ingieren alcohol } \\
\hline $\mathrm{Si}$ & (6) 16.7 & (4) 19.0 & (2) 11.1 & \multirow{2}{*}{0.78} \\
\hline No & (30) 83.3 & (17) 81.0 & (16) 88.9 & \\
\hline \multicolumn{5}{|l|}{ Dieta alta en grasas } \\
\hline $\mathrm{Si}$ & (9) 25.0 & (8) 38.1 & (6) 33.3 & \multirow{2}{*}{0.56} \\
\hline No & (27) 75.0 & (13) 61.9 & (12) 66.7 & \\
\hline \multicolumn{5}{|l|}{ Estrés moderado a alto } \\
\hline $\mathrm{Si}$ & (24) 66.7 & (13) 61.9 & (8) 44.4 & \multirow{2}{*}{0.28} \\
\hline No & (12) 33.3 & (8) 38.1 & (10) 55.6 & \\
\hline
\end{tabular}

Leyenda: BA - Breve asesoramiento; IPEF-USB - Indicación para la práctica de ejercicio físico en unidades de salud básicas; GE - Grupo de evaluación; ENT - Enfermedades Crónicas no Transmisibles; $p<0,05$

\section{AVAC}

Los valores medios al cabo de un año de intervención totalizaron para la estrategia GE en 0.788 , seguida de la intervención IPEF-USB con 0.791 y el BA sumando 0.800 AVAC. 
Costos de las Intervenciones

La estrategia GE costó \$15.32/persona/año, el BA presentó valor de \$16.26/persona/año y la estrategia IPEF-USB costó \$25.44/persona/año.

\section{RCEI}

La estrategia del BA fue más barata y obtuvo mayor AVAC al ser comparada con las demás intervenciones. En términos de AVAC ganados, la diferencia mayor se concentró entre la intervención BA vs. la intervención GE con un valor de 0.012 AVAC.

La Tabla 3 presenta el RCEI, siendo que el BA vs. GE presentó menor RCEI, por valor de \$78.33/AVAC. EI RCEI entre el BA vs. IPEF-USB obtuvo un ahorro de \$-1.020,00/AVAC.

Tabla 3. Diferencia de los costos, diferencia entre los AVAC y el RCEI entre las intervenciones $(n=75$, Rio Claro - SP)

\begin{tabular}{lccc}
\hline \multicolumn{1}{c}{ Comparaciones } & $\boldsymbol{\Delta}$ de los Costos & $\boldsymbol{\Delta}$ de los AVAC & RCEI \\
\hline BA vs. GE & $\$ 0.95$ & 0.012 & $\$ 78.33 /$ AVAC \\
IPEF-USB vs. GE & $\$ 10.13$ & 0.003 & $\$ 3.373,33 /$ AVAC \\
BA vs. IPEF-USB & $\$-9.18$ & 0.009 & $\$-1.020,00 /$ AVAC \\
\hline
\end{tabular}

Leyenda: AVAC - años de vida ajustados por calidad; BA - Breve asesoramiento; IPEF-USB Indicación para el ejercicio físico en unidades de salud básicas; GE - Grupo de evaluación; $\Delta$ - delta para identificar diferencias entre costos y AVAC

Actividad física

La figura 1 indica el porcentaje de usuarios activos físicamente en el tiempo libre, transporte y tiempo total (tiempo libre, transporte, trabajo y medio doméstico).

En relación con el porcentaje de participantes activos en el tiempo libre, no hubo diferencias estadísticamente significativas intragrupos y entre los grupos (Imagen 1 - A). Se encontró una diferencia estadísticamente significativa a los 12 meses entre breve asesoramiento (36.1\%) y grupo de evaluación $(11.1 \%)$ para los transportes de actividad física (Imagen 1 - B).

El grupo breve asesoramiento (94.4\%) presentó valores más altos que grupo de evaluación (66.7\%) a los 9 y 12 meses para la actividad física total (Imagen 1 C). Desde el 6 mes hasta el 12, el grupo de evaluación tuvo una disminución en el porcentaje de actividad física, mientras que el grupo breve asesoramiento se mantuvo hasta el $12^{\circ}$ mes. 


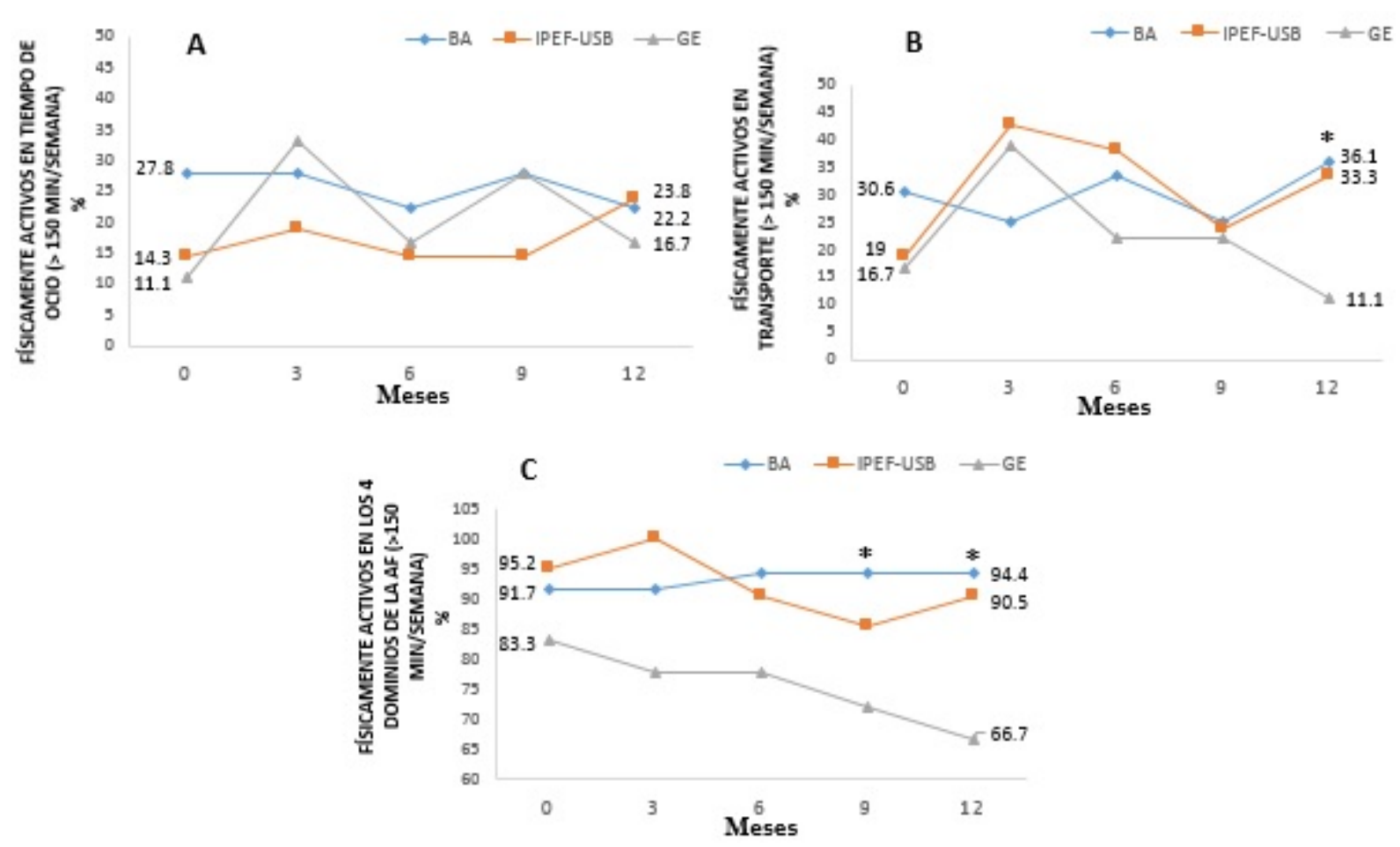

Figura 1. Prueba de chi cuadrado y porcentaje de físicamente activos en el ocio, en el transporte y tiempo total (Rio Claro - SP, $\mathrm{n}=75$ )

Leyenda: BA - Breve asesoramiento; IPEF-USB - Indicación para la práctica de ejercicio físico en unidades de salud básicas; GE - Grupo de evaluación; * significancia estadística para $p$ $<0,05$

\section{DISCUSIÓN}

Este estudio demostró que la estrategia de BA presentó un menor costo por AVAC ganado en comparación con GE y el grupo IPEF-USB. Además, el BA fue más efectivo para aumentar el número de personas físicamente activas en el dominio del transporte y el tiempo total en comparación con las otras estrategias, sin embargo, ninguna de las estrategias fue capaz de aumentar el porcentaje de personas físicamente activas en el tiempo libre.

Los resultados de costo-utilidad presentados en este estudio están en línea con los hallazgos de la literatura científica internacional, donde BA es más rentable que otras intervenciones. En la investigación realizara Vijay et al. $(2015)^{7}$, se encontraron que las intervenciones breves para promover la actividad física son rentables para la Atención Primaria de Salud en comparación con la atención habitual, incluso con la gran variación de los costos por AVAC obtenidos entre los países analizados, con valores de $\$ 75.49$ a $\$ 18.544,25$ por AVAC ganado.

Aun así, Bailly et al. (2018) ${ }^{21}$ compararon una intervención de ejercicio físico supervisado con una estrategia progresivamente autónoma, compuesta por pautas verbales y escritas sobre la actividad física para pacientes con enfermedades cardiovasculares, por lo que encontraron que ambas intervenciones fueron eficientes para reducir la atención médica después de un año; sin embargo, la intervención progresivamente autónoma fue más eficiente para mejorar los valores utilitarios y la calidad de vida de los participantes. Por lo 
anterior, Isaacs et al. (2007) ${ }^{22}$ identificaron que la estrategia de asesoramiento personalizado con el uso de materiales fue eficaz para aumentar el tiempo de actividad física y, en comparación con la estrategia de ejercicio físico supervisado y para el grupo de caminatas con supervisor, además, fue más económica.

Aún que las intervenciones de IPEF-USB y GE presentan valores de utilidad similares a los de BA, la diferencia a favor de BA en este estudio es el menor costo por persona para la atención anual; ya que el contacto del profesional de educación física con el usuario de Atención Primaria de Salud ocurrió en cinco momentos durante el año, mientras que el programa de ejercicios en Unidades de Salud Básicas requiere contacto dos veces por semana, lo que aumenta los costos y no favorece el costo-utilidad en comparación con BA.

Con respecto a la variable actividad física, los estudios de intervención realizados en países desarrollados con la estrategia BA y algunos realizados en Brasil encontraron resultados positivos en la calidad de vida, marcadores de salud (reducción de la adiposidad, presión arterial sistólica y diastólica, colesterol total) y tiempo de actividad física o mejoría en el porcentaje de actividad física como en este estudio $23,24,25,26,27,28$.

En este estudio, la actividad física recreativa no presentó diferencias estadísticamente significativas en el porcentaje de participantes físicamente activos, un hecho que puede justificarse porque todos los grupos alcanzaron y superaron las recomendaciones de actividad física ( $>150 \mathrm{~min} / \mathrm{semana}$ ) en el dominio total. De acuerdo con la revisión sistemática de Patnode et al. (2017) ${ }^{25}$, los autores identificaron que los estudios que incluían participantes con niveles sub-óptimos de actividad física al inicio del estudio (por debajo de 150 $\mathrm{min} / \mathrm{semana}$ ) dieron lugar a mayores aumentos en la actividad física en comparación con los que alcanzaron la recomendación de actividad física, como en este estudio.

Por otro lado, la mayoría de los estudios similares al desarrollado, se concentran en países desarrollados y con muestras que contienen un mayor número de participantes, colaborando con el poder de las pruebas estadísticas adoptadas; sin embargo, los resultados identificaron un pequeño porcentaje de participantes físicamente activos en el uso de su tiempo libre, relación con la estrategia de BA en comparación con la condición de no recibir intervención para promover la actividad física como se identificó en los estudios de Anokye et al. (2014) ${ }^{29}$, Leung et al. (2012) $)^{30}$ y Over et al. (2012) ${ }^{31}$.

Diferente del dominio del uso adecuado del tiempo libre, BA mostró niveles más altos de actividad física en el dominio de transporte en comparación con GE. Este hecho puede estar justificado porque la asesoría fomenta la práctica de actividad física en el transporte, contribuyendo a que el individuo comprenda que la locomoción activa es una estrategia importante para obtener beneficios para la salud. Además, la ciudad de Rio Claro (São Paulo, Brasil), tiene $32.79 \mathrm{~km}$ de carriles de ciclorruta para bicicletas ${ }^{32}$ y la muestra de este estudio es de bajos ingresos y trabaja fuera del hogar, por lo que tales condiciones pueden fomentar el transporte activo ${ }^{33}$ junto con los incentivos de BA. 
También se observó un aumento significativo en el porcentaje de actividad física en el tiempo total (suma de los cuatro dominios de la actividad física) para el BA en comparación con la GE (sin directrices sobre la actividad física) en el noveno y duodécimo mes, hubo mantenimiento para el BA y baja para el GE a partir del sexto mes, lo que indica una influencia positiva de los contenidos educativos de asesoramiento breve sobre sus participantes. De esta manera se genera afinidad por el estudio realizado por Fukuoka et al. (2019) ${ }^{34}$ reclutaron mujeres físicamente inactivas de la comunidad, con un promedio de 52 años y compararon diferentes intervenciones, incluyendo el uso de asesoramiento individual breve sobre la actividad física durante tres meses, con seguimiento durante los siguientes 6 meses utilizando el acelerómetro versus la intervención de control, que no recibió orientación sobre actividad física. El estudio indicó un aumento en el número de pasos por día y por hora, el incremento de actividad física moderada, vigorosa, moderado a vigoroso, evaluada por acelerómetro y actividad física autoinformada en los últimos 7 días después de la intervención. De esta forma, los resultados se mantuvieron exceptuando la actividad física autoinformada.

Por lo tanto, asociar la condición positiva de actividad física con el costo más bajo por AVAC ganado, fomenta la aplicabilidad de BA en Atención Primaria de Salud, especialmente dentro de la Unidades de Salud Básicas del Sistema Único de Salud de Brasil.

\section{CONCLUSIONES}

El estudio permite concluir que el BA y la estrategia de indicar al programa de ejercicio físico supervisado son estrategias adecuadas para mantener los niveles de actividad física durante un año; sin embargo, el BA presentó el menor costo por persona, mejor ganancia de AVAC/costo y porcentaje de actividad física en el dominio de transporte y el tiempo total en comparación con GE (condición de no recibir guía sobre actividad física), después de un año de intervención. Por lo tanto, se general a recomendación para el uso de la estrategia de BA como una posibilidad económicamente efectiva para la promoción de la salud y la Atención Primaria de Salud requiere de un professional de la educación física, por lo que se sugiere indicar la práctica supervisada de ejercicio físico con la asociación de BA.

Por lo tanto, los resultados encontrados en este estudio deben ser considerados para la toma de decisiones por parte de los gerentes de salud, sobre el incentivo para promover la actividad física en la Atención Primaria de Salud.

\section{REFERENCIAS BIBLIOGRÁFICAS}

1. Hallal PC, Andersen LB, Bull FC, Guthold R, Haske W, Ekelund U. Global physical activity levels: surveillance progress, pitfalls, and prospects. The Lancet. 2012; 380 (9838): 247-257. https://doi.org/10.1016/S0140-6736(12)60646-1

2. Vigilância de Fatores de Risco e Proteção para Doenças Crônicas por Inquérito Telefônico (VIGITEL BRASIL). Estimativas sobre frequência e distribuição 
sociodemográficas de fatores de risco e proteção para doenças crônicas nas capitais dos 26 estados brasileiros e no distrito federal em 2016. Brasília: Ministério da Saúde; 2016.

3. Reis RS, Salvo D, Ogilvie D, Lambert EV, Goenka S, Brownson RC. Scaling up physical activity interventions worldwide: stepping up to larger and smarter approaches to get people moving. Lancet. 2016; 388 (10051):1337-48. https://doi.org/10.1016/S0140-6736(16)30728-0

4. Becker LA, Gonçalves PB, Reis RS. Programas de promoção da atividade física no Sistema Único de Saúde brasileiro: revisão sistemática. Rev Bras Ativ Fís \& Saúde. 2016; 21 (2): 110-122. https://doi.org/10.12820/rbafs.v.21n2p110-122

5. Rascati KL. Introdução à Farmacoeconomia. Análise de Custo-Utilidade. $1^{\circ}$ Edição. Artemed, 2010.

6. Garrett S, Elley CR, Rose BS, O'Dea DAB, Dowell CA. Are physical activity interventions in primary care and the community cost-effective? A systematic review of the evidence. $\mathrm{Br} J$ Gen Pract. 2011; 61 (584): 125-133. https://doi.org/10.3399/bjgp11X561249

7. Vijay GC, Wilson EC, Suhrcke M, Hardeman W, Sutton S. Are brief interventions to increase physical activity cost-effective? A systematic review. $\mathrm{Br} J$ Sports Med. 2016: 50 (7): 408-417. https://doi.org/10.1136/bjsports-2015-094655

8. NICE. Four commonly used methods to increase physical activity. 2006. Available in:<http://guidance.nice.org.uk/ph2>.

9. West D, Saffin K. Literature review: Brief interventions and childhood obesity for North West and London Teaching Public Health Networks. Public Health Resource Unit. 2008.

10. Ministério da Saúde. Secretaria de Vigilância em Saúde. Secretaria de Atenção à Saúde. Política Nacional de Promoção da Saúde/Ministério da Saúde, Secretaria de Vigilância em Saúde, Secretaria de Atenção à Saúde. 3th ed. Brasília: Ministério da Saúde. 2010.

11. Santos RP, Horta PM, Souza CS, Santos CA, Oliveira HBS, Almeida LMR, Santos LC. Aconselhamento sobre alimentação e atividade física: prática e adesão de usuários da atenção primária. Rev Gaúcha Enferm. 2012; 33 (4). https://doi.org/10.1590/S1983-14472012000400002

12. Lopes ACS, Toledo MTT, Câmara AMCS, Menzel HJK, Santos LC. Condições de saúde e aconselhamento sobre alimentação e atividade física na Atenção Primária à Saúde de Belo Horizonte-MG. Epidemiol Serv Saúde. 2014; 23 (3). https://doi.org/10.5123/S1679-49742014000300010

13. Häfele V, Siqueira F. Aconselhamento para atividade física e mudança de comportamento em Unidades Básicas de Saúde. Rev Bras de Ativ Fís \& Saúde. 2016; 21(6). https://doi.org/10.12820/rbafs.v.21n6p581-592

14. Campos L. Análise do custo de intervenções para promoção de atividade física em unidades de saúde da família de Rio Claro-SP [dissertação]. Rio Claro: UNESP; 2017.

15. Nakamura PM, Papini CB, Chiyoda A, Gomes GAO, Netto AV, Teixeira IP, Luciano $E$, Kokubun E. Programa de intervenção para a prática de atividade física: Saúde Ativa Rio Claro. Rev Bras de Ativ Fís \& Saúde. 2010; 15 (2): 128132. https://doi.org/10.12820/rbafs.v.15n2p128-132

16. Ciconelli MR, Ferraz BM, Santos W, Meinão I, Quaresma RM. Tradução para a língua portuguesa e validação do questionário genérico de avaliação de qualidade de vida SF-36 (Brasil SF-36)/Brazilian-Portuguese version of the SF- 
36. A reliable and valid quality of life outcome measure. Rev Bras Reumatologia. 1999; 39 (3): 143-150.

17. Cruz LN, Camey SA, Hoffmann JF, Rowen D, Brazier JE, Fleck MP, Polanczyk CA. Estimating the SF-6D value set for a population based sample of Brazilians. Value Health. 2011; 14 (5): 108-14. https://doi.org/10.1016/j.jval.2011.05.012

18. Sposito LAC. Análise econômica de custo-utilidade de três intervenções para promoção de atividade física em usuários do SUS de Rio Claro - SP [dissertação]. Rio Claro: UNESP, 2017.

19. Drummond MF, Sulpher MJ, Torrance GW, O'Brien BJ, Stoddart GL. Methods for the Economic Evalution of Health Care Programmes. $3^{\circ}$ Edição. Hampshire: Oxford University Press, 2005.

20. Hallal PC, Victora CG, Wells JC, Lima RC. Physical inactivity: prevalence and associated variables in Brazilian adults. Medicine \& Science in Sports \& Exercise. $2003 ; \quad 35 \quad$ (11): https://doi.org/10.1249/01.MSS.0000093615.33774.0E

21. Bailly L, Mossé P, Diagana S, Fournier M, d'Arripe-Longueville F, Diagana O, Gal J, Grebet J, Moncada M, Domerego JJ, Radel R, Fabre R, Fuch A, Pradier C. "As du Coeur" study: a randomized controlled trial on quality of life impact and cost effectiveness of a physical activity program in patients with cardiovascular disease. BMC Cardiovasc Disord. 2018; 18: 225. https://doi.org/10.1186/s12872-018-0973-3

22. Isaacs A, Critchley J, Tai SS, Buckingham K, Westley D, Harridge SDR, Smith C, Gottlieb JM. Exercise Evaluation Randomised Trial (EXERT): a randomised trial comparing GP referral for leisure centre-based exercise, community-based walking and advice only. Health Technol Assess. 2007; 11 (10): 1-165.

https://doi.org/10.3310/hta11100

23. Elley C, Kerse N, Arroll B, Robinson E. Effectiveness of counselling patients on physical activity in general practice: cluster randomised controlled trial. BMJ. 2003; 326 (7393), p.793-793. https://doi.org/10.1136/bmj.326.7393.793

24. Lin JS, O'Connor E, Whitlock EP, Beil TL. Behavioral Counseling to Promote Physical Activity and a Healthful Diet to Prevent Cardiovascular Disease in Adults: A Systematic Review for the U.S. Preventive Services Task Force. Ann Intern Med. 2010; 153 (11): 736-750. https://doi.org/10.7326/0003-4819-15311-201012070-00007

25. Patnode C, Evans C, Senger C, Redmond N, Lin J. Behavioral Counseling to Promote a Healthful Diet and Physical Activity for Cardiovascular Disease Prevention in Adults Without Known Cardiovascular Disease Risk Factors. JAMA. 2017; 318 (2): 175-193. https://doi.org/10.1001/jama.2017.3303

26. Gomes MA, Duarte MFS. Efetividade de uma intervenção de atividade física em adultos atendidos pela estratégia saúde da família: Programa Ação e Saúde Floripa - Brasil. Rev Bras de Ativ Fís \& Saúde. 2008; 13 (1).

http://dx.doi.org/10.12820/rbafs.v.13n1p44-56

27. Benedetti TRB, Schwingel A, Gomez LSR, Chodzko-Zajko W. Programa "VAMOS" (Vida Ativa Melhorando a Saúde): da concepção aos primeiros resultados. Rev Bras de Cineantropometria e Desempenho Humano. 2012; 14 (6): 723-737. https://doi.org/10.5007/1980-0037.2012v14n6p723

28. Ribeiro E, Garcia L, Salvador E, Costa E, Andrade D, Latorre M, Florindo A. Assessment of the effectiveness of physical activity interventions in the Brazilian Unified Health System. Rev Saúde Pública. 2017; 26: 51-56. 


\section{https://doi.org/10.1590/s1518-8787.2017051006654}

29. Anokye NK, Lord J, Fox-Rushby J. Is brief advice in primary care a cost-effective way to promote physical activity. Br J Sports Med. 2014; 48: 202-206. https://doi.org/10.1136/bjsports-2013-092897

30. Leung W, Ashton T, Kolt GS, Schofield GM, Garrett N, Kerse N, Patel A. Costeffectiveness of pedometer-based versus time-based Green Prescriptions: The Healthy Steps Study. Australian Journal of Primary Health. 2012; 18: 204-211. https://doi.org/10.1071/PY11028

31. Over EAB, Wendel-Vos GCW, Berg M, Reenen HHH, Tariq L, Hoogenveen R, Baal PHM. Cost-effectiveness of counseling and pedometer use to increase physical activity in the Netherlands: a modeling study. Cost Effectiveness and Resource Allocation. 2012; 10 (13). https://doi.org/10.1186/1478-7547-10-13

32. Teixeira IP. Impacto da implementação de ciclofaixas na utilização da bicicleta como meio de transporte [tese]. Rio Claro - SP: UNESP, 2016.

33. Sá TH, Pereira RHM, Duran AC, Monteiro CA. Diferenças socioeconômicas e regionais na prática do deslocamento ativo no Brasil. Rev Saúde Pública. 2016; 50:(37). https://doi.org/10.1590/S1518-8787.2016050006126

34. Fukuoka Y, Haskell W, Lin F, Vittinghoff E. Short- and Long-term Effects of a Mobile Phone App in Conjunction With Brief In-Person Counseling on Physical Activity Among Physically Inactive Women: The mPED Randomized Clinical Trial. JAMA Netw Open. 2019; 2 (5):1-13. https://doi.org/10.1001/jamanetworkopen.2019.4281

Número de citas totales/Total references: 34

Número de citas propias de la revista / Journal's own references: 0

Rev.int.med.cienc.act.fís.deporte - vol. 21 - número 83 - ISSN: 1577-0354 